\title{
Seasonal and Geographical Variation of Dengue Vectors in Narathiwat, South Thailand
}

\author{
Ornanong Boonklong ${ }^{1}$ and Adisak Bhumiratana ${ }^{2}$ \\ ${ }^{1}$ Department of Mathematics and Statistics, Faculty of Science and Technology, Nakhon Si Thammarat Rajabhat University, \\ Muang, Nakhon Si Thammarat 80280, Thailand \\ ${ }^{2}$ Center of Ecohealth Education and Research (CEER), Faculty of Public Health, Thammasat University, Rangsit Campus, \\ Pathumthani 12121, Thailand \\ Correspondence should be addressed to Ornanong Boonklong; bkornanong@gmail.com
}

Received 28 February 2016; Revised 17 May 2016; Accepted 31 May 2016

Academic Editor: Julia Uhanova

Copyright ( 2016 O. Boonklong and A. Bhumiratana. This is an open access article distributed under the Creative Commons Attribution License, which permits unrestricted use, distribution, and reproduction in any medium, provided the original work is properly cited.

\begin{abstract}
Using GIS-based land use map for the urban-rural division (the relative ratio of population density adjusted to relatively Aedesinfested land area), we demonstrated significant independent observations of seasonal and geographical variation of Aedes aegypti and Aedes albopictus vectors between Muang Narathiwat district (urban setting) and neighbor districts (rural setting) of Narathiwat, Southern Thailand, based on binomial distribution of Aedes vectors in water-holding containers (water storage containers, discarded receptacles, miscellaneous containers, and natural containers). The distribution of Aedes vectors was influenced seasonally by breeding outdoors rather than indoors in all 4 containers. Accordingly, both urban and rural settings elicited significantly seasonal (wet versus dry) distributions of $A$ e. aegypti larvae observed in water storage containers $(P=0.001$ and $P=0.002)$ and natural containers $(P=0.016$ and $P=0.015)$, whereas, in rural setting, the significant difference was observed in discarded receptacles $(P=0.028)$ and miscellaneous containers $(P<0.001)$. Seasonal distribution of Ae. albopictus larvae in any containers in urban setting was not remarkably noticed, whereas, in rural setting, the significant difference was observed in water storage containers $(P=0.007)$ and discarded receptacles $(P<0.001)$. Moreover, the distributions of percentages of container index for Aedes-infested households in dry season were significantly lower than that in other wet seasons, $P=0.034$ for urban setting and $P=0.001$ for rural setting. Findings suggest that seasonal and geographical variation of Aedes vectors affect the infestation in those containers in human inhabitations and surroundings.
\end{abstract}

\section{Introduction}

Dengue vectors responsible for transmission of any dengue virus serotype (DENV 1 to 4 ) can infest or reinfest geographically widespread areas of human inhabitations around the globe [1-5], and their breeding characteristics are diverse [4, $6,7]$. The dynamics of dengue vector ecology is constrained seasonally and geographically by ecological relationships in nature. In Southeast Asia including Thailand, two common anthropophagic dengue vectors, namely, Aedes (Stegomyia) aegypti (Linnaeus) and Aedes albopictus (Skuse), are adapted well to local environments, although the environments favorable to their infestation or reinfestation relate breeding characteristics to human settlements and activities [6-13]. As for the vectorial capacity, Ae. aegypti rather than Ae. albopictus has been responsible for transmission of any DENV serotype in urban settings rather than in rural settings. Ae. aegypti serves as primary dengue vector and plays significant role in vertical and transovarial dengue transmission of urban cycle [14-16].

Dengue risk estimates that the projections are strongly associated with climatic variables (temperature, precipitation, and humidity) and socioeconomic factors (population size, population density, and gross domestic product or GDP per capita) and have been shown in a warmer world for the spread of dengue in certain transmission areas or prone areas due to complex interactions of climate changes-whether globally, regionally, or locally-and socioeconomic development [1720]. Also, the estimated risks of increase in dengue incidence 
are driven geographically and seasonally by increasing the proportions of the population in the urban and rural areas that have access to domestic water supply systems whether the availability of piped water or water storage tanks $[17,18]$. However, most dengue risk estimates rely on nonlinearity of seasonal and geographical distributions of dengue incidence rather than dengue vectors adapted to local environments or present in receptive areas.

In Thailand, dengue vector control focuses mainly on containment of Aedes breeding places in the impoverished human inhabitations at which water-holding containers whether artificial or natural are abandoned in buildings and public spaces [21]. But common obstacles have been the results from the improper directions of the dengue vector surveillance and control to specific target populations and the lack of effective and sustained environmental management at household and community levels [22-24]. Regarding this, any surveyed households or containers infested with $A e$. aegypti are always used as the units for dengue vector surveillance whether because the degree of the infestation (house index, HI or container index, and CI) is demarcated by the stratification of dengue transmission risk or the reduction of the infestation level is achieved by dengue vector control.

Moreover, the complex interactions of driving factors such as agricultural land use changes, unplanned urbanization, and physical environmental conditions $[14,18,20,25$, 26] are considered the linkages of geographical distributions of Aedes vectors that can infest or reinfest in receptive areas. This infers the degree to which dengue transmission risk occurs in certain transmission areas or especially in prone areas. For instance, in the 2000s, several reports demonstrated that dengue transmission risks in urban areas of Thailand have become increasingly evident $[8,15,23-26]$, as the urban-rural gradient pertaining to seasonal and geographical distribution of Aedes infestation remains to be established. To address this issue, the study therefore focused on land use/land cover changes; that is, land areas geographically defined in landscape structure in which human activities such as socioeconomic development, settlements/resettlements, and outdoor activities can induce changes in land use types such as urban and built-up land and agricultural land. This conceptual novelty makes the constructive urban-rural division by making use of geographical information systems(GIS-) based land use map.

The study objective was to determine seasonal and geographical variation of dengue vectors that infested in waterholding containers in different settings of Narathiwat, Southern Thailand, where the urban-rural gradient discriminates between Muang Narathiwat district (as urban setting) and neighbor districts (as rural setting). The abundance and distribution of Aedes vectors that infested in different containers, as well as in households of the urban and rural settings, were compared.

\section{Materials and Methods}

2.1. Study Area and Design. The study area that was confined to Narathiwat province, Southern Thailand, covered three districts, namely, Muang Narathiwat, Ra-ngae, and Choairong (Figure 1). Based on the urban-rural division, the heterogeneity of Aedes-infested land area is influenced by the pattern and extent of urban and built-up land area whether high or low density of the population. There might exist diverse breeding characteristics by two different urban and rural climates of human inhabitations connecting to vegetation such as orchards mixed or not mixed with other perennial trees such as rubber trees and oil palms $[6,8,15,25$, 26]. Thus, the relative ratio of population density adjusted to the relatively Aedes-infested land area of the selected district was used in this study.

With respect to the administrative level of district, the population number is a numerator and the total land area or the relatively Aedes-infested land area is a denominator. The relative population density ratio (PDR) for a given district is mathematically expressed as the population density adjusted to the relatively Aedes-infested land area (population number/the urban and built-up land area connecting to vegetation in $\mathrm{km}^{2}$ ) divided by the population density (population number/total land area in $\mathrm{km}^{2}$ ). Muang Narathiwat had a population of 115,847 (of 2012 midyear), total land area of $299.218 \mathrm{~km}^{2}$, and Aedes-infested land area of $121.293 \mathrm{~km}^{2}$. Rangae had a population of 76,817 , total land area of $435.581 \mathrm{~km}^{2}$, and Aedes-infested land area of $225.893 \mathrm{~km}^{2}$. Cho-airong had a population of 37,790 , total land area of $185.07 \mathrm{~km}^{2}$, and Aedes-infested land area of $103.941 \mathrm{~km}^{2}$. Hence, we obtained the PDR for Muang Narathiwat district $(\mathrm{PDR}=2.467)$ and two neighbor districts: Cho-airong $(\mathrm{PDR}=1.780)$ and $\mathrm{Ra}-$ ngae $(\mathrm{PDR}=1.928)$ (Figure 1$)$.

The PDR value for each district was considered as the parameter that infers difference in the spatial distributions of Aedes vectors between the urban and rural settings. Only the season variation was considered as temporal distribution of Aedes infestation. This permitted a stratified cluster random sampling of total 300 houses (150 houses for each study setting); all of which were georeferenced and monitored to determine whether any household and any water-holding containers were infested with Ae. aegypti or Ae. albopictus, between wet and dry seasons. Both households and containers were used as the unit of analysis throughout the study.

2.2. Aedes Larval Survey and Monitoring. As mentioned above, the entomological surveys were conducted between the wet (November-December 2012) and dry (March-April 2013) seasons, based on the baseline meteorological data such as monthly rainfall and rainfall days (Figure 2). Mean monthly rainfall and rainfall days in 2012-2014 wet season are $687.15 \mathrm{~mm}$ and 21 days, whereas, in 2012-2014 dry season, there were $123.7 \mathrm{~mm}$ and 7.5 days. Prior to the Aedes larval survey in 2012, two entomological survey teams including the authors were trained in how to standardize qualitatively the cluster random sampling of houses as well as in how to observe, collect any larva or pupa found in any waterholding containers, and record the information of any waterholding container type, whether artificial or natural, found in any household as described below. Because environmental 


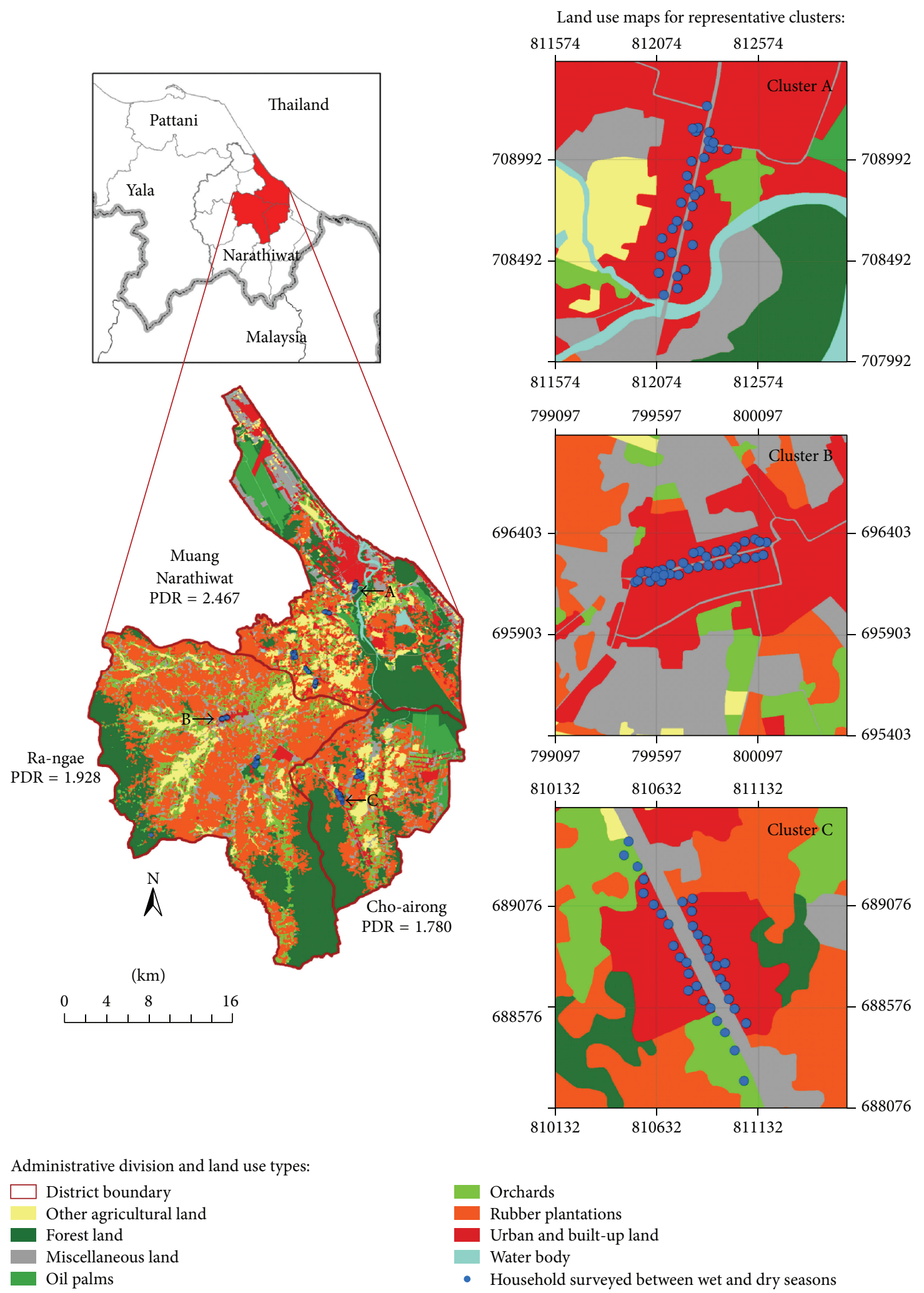

FIGURE 1: GIS-based land use map illustrated with two related urban and rural settings of Narathiwat. The relative population density ratio (PDR) for each district is shown to discriminate the urban-rural gradient. This approach was consistent with the degree of urbanization [9]. All 300 georeferenced houses that were used to conduct Aedes larval surveys were derived from 5 clusters of Muang Narathiwat (30 houses each), 2 clusters of Ra-ngae ( 40 and 35 houses), and 2 clusters of Cho-airong ( 40 and 35 houses). Representative cluster for each district that was assigned to a grid of $500 \mathrm{~m}^{2}$ is shown. Miscellaneous land included roads, landfills, pits (soil, sand, and laterite), scrubs, bamboos, marsh, swamp, grass, and others. All the land use maps that were also validated by the ground surveys between 2012 and 2013 were constructed using the ArcGIS ver 10.1 software applications. 


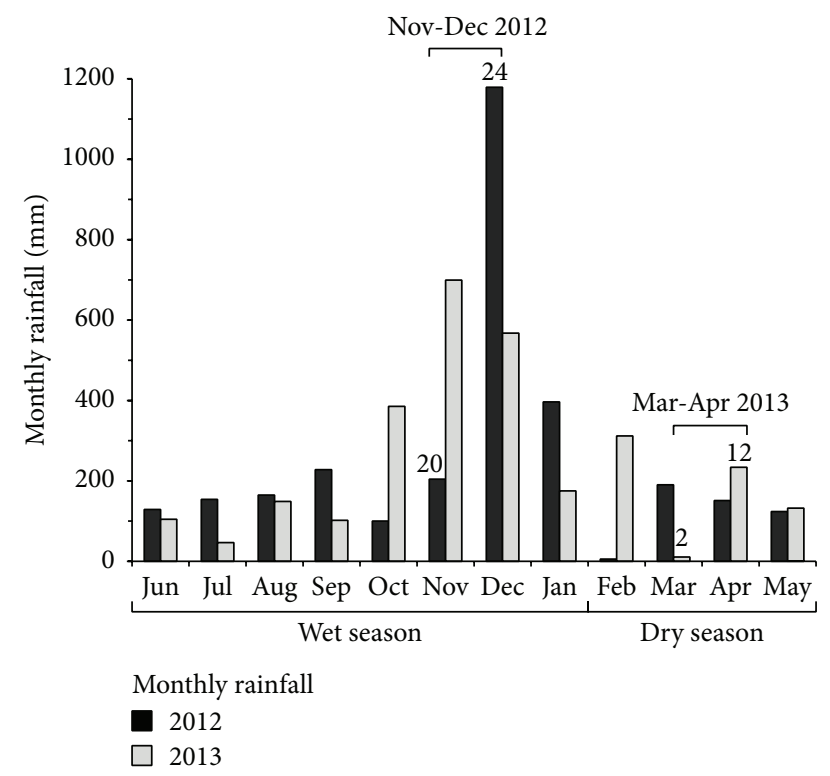

FIGURE 2: Monthly rainfall data for a period of two consecutive years, 2012-2013. Difference in the monthly rainfall (millimeters) was observed during which the Aedes larval surveys were conducted between wet season (November-December 2012) and dry season (March-April 2013). Also, the numberings inside and outside the bars indicate rainfall days that were observed during the studied months of wet season, November (20) and December (24), and of dry season, March (2) and April (12), respectively. These meteorological data were obtained from the Narathiwat Meteorological Station.

cleaning practices at household level $[15,21-23]$ might influence the indoor and outdoor distributions of water-holding containers whether or not they yielded larva productivity in any season, the surveyed containers that did not contain water were not recorded. All the surveyed households were georeferenced, and photographic evidence was recorded to compare whether the indoor and outdoor distributions of water-holding containers found between two seasons were the same. In any positive water-holding container, any larva or pupa stage of Ae. aegypti or Ae. albopictus initially monitored using the flashlight was collected, placed into a $200 \mathrm{~mL}$ plastic cup with cover [9], and then transferred to the laboratory. Then, pictorial keys for the identification of Ae. aegypti or Ae. albopictus [27] were used to taxonomically examine any larva found in any positive container by the entomological experts.

2.3. Water-Holding Container-Level Information. Regarded as breeding places for Ae. aegypti and Ae. albopictus, both artificial and natural water-holding containers were monitored, during the day time, inside and outside the houses as mentioned earlier. The indoor location was the place at which any water-holding container was found inside the house. The outdoor location was the place at which any water-holding container was found under the roof or within a radius of 5-10 meters of the premise [6]. The artificial water-holding containers were initially recorded as types and materials. Then they were categorized into 3 types of containers: water storage containers including small to large earthen jars, cement tanks, plastic drums, or bath basins; discarded receptacles including used tyres, bottles, cans, ice bins, buckets, boxes, boats, or cars; miscellaneous containers including flowerpots, flower vessels, saucers, refrigerator drain pans, cabinet ant traps, or cup/bowl/bottle water feeders [21]. Other natural containers were also recorded as types such as coconut shells, bamboo stumps, tree holes, and leaf axils [21]. Larva or pupa collection from any water-holding container was done between 0800 and $1700 \mathrm{~h}$ as described earlier.

Regardless of the kind of Aedes vectors, the container index (CI) was used to describe seasonal distributions of the Aedes infestation in container types between urban and rural settings. The CI value that infers the Aedes larva/pupaproducing container found in any surveyed household in any season was derived by calculating the number of any positive containers in total of surveyed water-holding containers, multiplied by 100 .

2.4. Household-Level Information. During Aedes larval survey in the urban or rural setting, any infested household was defined as having a water-holding container that contained at least one larva or pupa stage of Ae. aegypti and/or Ae. albopictus. The number of Aedes-infested households was further analyzed temporally and spatially for distributions of Aedes vectors between two study settings. The house index (HI, \%) was used to describe seasonal distributions of the Aedes infestation in receptive households between urban and rural settings. The HI value infers the number of receptive households that produce any positive containers with any Aedes vectors seasonally in the study setting. The HI (\%) was derived by calculating the number of positive households, whether infested with Ae. aegypti, Ae. albopictus, or both in total of surveyed households, multiplied by 100 .

2.5. Seasonal and Geographical Analysis of Aedes Infestation. Seasonal and geographical analysis of Aedes vectors that infested in different container types was based on the assumption of binomial distribution of the Aedes vectors that infested in two related urban and rural settings of Narathiwat; that is, any larvae of Ae. aegypti or Ae. albopictus, or both were found in any water-holding containers inside or outside the houses between wet and dry seasons. In this study, none or a very small number of the pupae found in all the containers were negligible. When using the container as the unit of analysis, McNemar's test was used to compare the proportions or test the difference between the proportions with two-sided $P$ value $<0.05$, by performing on $2 \times 2$ contingency table with two discrete dichotomous variables. The null hypothesis of marginal homogeneity is an equal distribution of waterholding containers, as well as Aedes-infested ones, between urban and rural settings whether they are distributed seasonally and domestically. As for the abundance of Aedes vectors, mean $( \pm 2$ standard errors or $\mathrm{SE})$ was presented to describe the larva numbers of Ae. aegypti or Ae. albopictus in containers observed between two seasons in the urban or rural settings of Narathiwat. The Mann-Whitney $U$ test $(P<0.05)$ was used to determine whether larva numbers of Ae. aegypti or Ae. albopictus found in each type of containers 
in wet season are lower or higher than that in the other dry season by comparing the mean ranks of each distribution of Aedes larvae. The null hypothesis is that the distributions are identical and the mean ranks are the same for twoindependent samples.

When using the household as the unit of analysis, $\mathrm{McNe}$ mar's test was used as before to compare the proportions of the cluster sample of households in the urban setting $(n=$ $150)$ or rural setting $(n=150)$ that were infested with Aedes vectors between two seasons. Because both urban and rural settings seemed to have similar distributions of Aedesinfested households, mean percentages $( \pm 2$ SE) of CI values for two-independent samples were presented. The mean CI (\%) that infers the distribution of positive containers in individual household infested with Aedes vectors was derived by calculating the percentage or proportion of positive containers in a total of water-holding containers found in an infested household in any season. The Mann-Whitney $U$ test $(P<0.05)$ was used as before to test the equal distribution of mean CI values independently observed between two seasons in two study settings.

\section{Results}

3.1. Water-Holding Container as the Unit of Analysis. A total of 4,441 water-holding containers observed in two seasons included 2,193 (49.4\%) containers from the urban setting and $2,248(50.6 \%)$ containers from the rural setting (Table 1). On the other hand, artificial containers (94\%) served as key containers, fifteenfold higher than natural containers (6\%), as shown in Table 1 . However, it was likely to show temporal and spatial distributions of water-holding containers whether artificial or natural. As for artificial container type, there was significant difference in the outdoor and indoor distributions of water-holding containers that were independently observed only in wet season between the urban and rural settings $(P=0.002)$. As for natural container type, there was significant difference in the outdoor and indoor distributions of water-holding containers that were independently observed in wet season between the urban and rural settings $(P<0.001)$. Meanwhile, all natural containers were found only indoors in dry season.

When analyzed for Aedes infestation as a result of the urban-rural division, Table 2 shows temporal and spatial distributions of Aedes vectors that infested in 4 container types regardless of the kind of Aedes vectors. Regardless of container type, the Aedes infestation level of $18.8 \%$ (835/4,441), CI: $9.6 \%(425 / 4,441)$ for wet season, and $9.2 \%$ $(410 / 4,441)$ for dry season was observed. The urban and rural settings differed significantly in the outdoor and indoor distributions of Aedes-infested water storage containers that were independently observed in both wet $(P<0.001)$ and dry $(P<0.001)$ seasons. Similarly, the urban and rural settings differed significantly in the outdoor and indoor distributions of Aedes-infested miscellaneous containers $(P=0.003)$, as well as Aedes-infested natural containers $(P<0.001)$; all were independently observed only in wet season. As for Aedesinfested discarded receptacle type, none was found indoors between two seasons in both study settings.
Based on these independent observations of Aedes vectors that infested in the water-holding containers but likely bred outdoors rather than indoors (Table 2), we omitted the likelihood that larval abundances of Aedes vectors were influenced by indoor breeding characteristics as the result of the urbanrural division in this empirical analysis (Figure 3). Because the pupa number found in any containers was negligible, only the abundance of Aedes vectors, that is, presented by mean larva numbers $( \pm 2 \mathrm{SE}$ ) of Ae. aegypti (Figure 3(a)) and Ae. albopictus (Figure 3(b)), was further analyzed by comparing the abundance of Ae. aegypti and Ae. albopictus in any outdoor containers of each container type that produced larvae between two seasons in two urban and rural settings.

In Figure 3(a), the urban setting elicited seasonal distributions (wet versus dry) of the Ae. aegypti-infested water storage containers ( $n=85$ versus 80$)$, discarded receptacles $(n=22$ versus 64$)$, miscellaneous containers ( $n=38$ versus 45$)$, and natural containers ( $n=20$ versus 26$)$. Seasonal distributions of Ae. aegypti larvae observed only in the water storage containers and natural containers were remarkably noticed. The distributions of Ae. aegypti larva numbers in water storage containers observed in dry season were significantly lower than that observed in other wet seasons $(Z=-3.252$, $P=0.001)$. The distributions of Ae. aegypti larva numbers in natural containers observed in dry season were significantly lower than that observed in other wet seasons $(Z=-2.410$, $P=0.016)$. Similar to the urban setting, the rural setting showed seasonal distributions (wet versus dry) of Ae. aegyptiinfested water storage containers ( $n=97$ versus 61), discarded receptacles $(n=15$ versus 57$)$, miscellaneous containers ( $n=118$ versus 49$)$, and natural containers ( $n=15$ versus 17) (Figure 3(a)). However, the rural settings contrasted seasonal distributions of Ae. aegypti larvae in all 4 container types. The distributions of Ae. aegypti larvae observed in dry season were significantly lower than that observed in other wet seasons: water storage containers $(Z=-3.040$, $P=0.002)$, discarded receptacles $(Z=-2.195, P=0.028)$, miscellaneous containers $(Z=-6.185, P<0.001)$, and natural containers $(Z=-2.425, P=0.015)$.

In Figure 3(b), the urban setting also elicited seasonal distributions (wet versus dry) of the Ae. albopictus-infested water storage containers ( $n=84$ versus 78$)$, discarded receptacles ( $n=22$ versus 60$)$, miscellaneous containers $(n=36$ versus 46 ), and natural containers ( $n=19$ versus 27 ). Unlike that of Ae. aegypti-infested containers, the seasonal distributions of Ae. albopictus larvae in any containers were not pronounced. Similar to the urban setting, the rural setting also exhibited seasonal distributions (wet versus dry) of $A e$. albopictus-infested water storage containers $(n=102$ versus 58 ), discarded receptacles ( $n=17$ versus 52$)$, miscellaneous containers ( $n=101$ versus 42$)$, and natural containers ( $n=14$ versus 15 ) (Figure 3(b)). Only seasonal distributions of Ae. albopictus larvae in the water storage containers and discarded receptacles were remarkably noticed. The distributions of Ae. albopictus larvae observed in dry season were significantly lower than that observed in wet season: water storage containers $(Z=-2.677, P=0.007)$ and discarded receptacles $(Z=-4.077, P<0.001)$. 
TABLE 1: Seasonal and geographical distributions of water-holding containers.

\begin{tabular}{|c|c|c|c|c|c|c|}
\hline \multirow[t]{2}{*}{ Container type } & \multirow[t]{2}{*}{ Season } & \multirow[t]{2}{*}{ Location } & \multicolumn{3}{|c|}{$\begin{array}{c}\text { Number (\%) of containers surveyed between the study } \\
\text { settings }\end{array}$} & \multirow[t]{2}{*}{$P$ value } \\
\hline & & & Urban & Rural & Total & \\
\hline \multirow{6}{*}{ Artificial } & \multirow{3}{*}{ Wet } & Outdoor & 383 & 404 & $787(41.1)$ & \multirow{3}{*}{$0.002^{*}$} \\
\hline & & Indoor & 496 & 633 & $1,129(58.9)$ & \\
\hline & & Total & $879(45.9)$ & $1,037(54.1)$ & 1,916 & \\
\hline & \multirow{3}{*}{ Dry } & Outdoor & 629 & 470 & $1,099(48.8)$ & \multirow{3}{*}{0.180} \\
\hline & & Indoor & 513 & 640 & $1,153(51.2)$ & \\
\hline & & Total & $1,142(50.7)$ & $1,110(49.3)$ & 2,252 & \\
\hline \multirow{6}{*}{ Natural } & \multirow{3}{*}{ Wet } & Outdoor & 46 & 40 & $86(97.7)$ & \multirow{3}{*}{$<0.001^{*}$} \\
\hline & & Indoor & 0 & 2 & $2(2.3)$ & \\
\hline & & Total & $46(68.1)$ & $42(47.9)$ & 88 & \\
\hline & \multirow{3}{*}{ Dry } & Outdoor & 126 & 59 & $185(100)$ & \multirow{3}{*}{ NA } \\
\hline & & Indoor & 0 & 0 & 0 & \\
\hline & & Total & $126(68.1)$ & 59 (31.9) & 185 & \\
\hline
\end{tabular}

NA: not applicable.

* Statistically significant with McNemar's test for two-independent samples.

TABLE 2: Seasonal and geographical distributions of Aedes vectors in water-holding containers.

\begin{tabular}{|c|c|c|c|c|c|c|}
\hline \multirow[t]{2}{*}{ Container type } & \multirow[t]{2}{*}{ Season } & \multirow[t]{2}{*}{ Location } & \multicolumn{3}{|c|}{$\begin{array}{c}\text { Number (\%) of positive containers surveyed between } \\
\text { the study settings }\end{array}$} & \multirow[t]{2}{*}{$P$ value } \\
\hline & & & Urban & Rural & Total & \\
\hline \multirow{6}{*}{ Water storage } & \multirow{3}{*}{ Wet } & Outdoor & 84 & 81 & $165(86.4)$ & \multirow{3}{*}{$<0.001^{*}$} \\
\hline & & Indoor & 4 & 22 & $26(13.6)$ & \\
\hline & & Total & $88(46.1)$ & $103(53.9)$ & 191 & \\
\hline & \multirow{3}{*}{ Dry } & Outdoor & 76 & 54 & $130(89.7)$ & \multirow{3}{*}{$<0.001^{*}$} \\
\hline & & Indoor & 8 & 7 & $15(10.3)$ & \\
\hline & & Total & $84(57.9)$ & $61(42.1)$ & 145 & \\
\hline \multirow{6}{*}{ Discarded receptacles } & \multirow{3}{*}{ Wet } & Outdoor & 22 & 17 & $39(100)$ & \multirow{3}{*}{ NA } \\
\hline & & Indoor & 0 & 0 & 0 & \\
\hline & & Total & $22(56.4)$ & $17(43.6)$ & 39 & \\
\hline & \multirow{3}{*}{ Dry } & Outdoor & 65 & 57 & $122(100)$ & \multirow{3}{*}{ NA } \\
\hline & & Indoor & 0 & 0 & 0 & \\
\hline & & Total & $65(53.3)$ & $57(46.7)$ & 122 & \\
\hline \multirow{6}{*}{ Miscellaneous containers } & \multirow{3}{*}{ Wet } & Outdoor & 15 & 52 & $67(41.9)$ & \multirow{3}{*}{$0.003^{*}$} \\
\hline & & Indoor & 25 & 68 & $93(58.1)$ & \\
\hline & & Total & $40(25.0)$ & $120(75.0)$ & 160 & \\
\hline & \multirow{3}{*}{ Dry } & Outdoor & 11 & 26 & $37(37.4)$ & \multirow{3}{*}{0.162} \\
\hline & & Indoor & 38 & 24 & $62(62.6)$ & \\
\hline & & Total & $49(49.5)$ & $50(50.5)$ & 99 & \\
\hline \multirow{6}{*}{ Natural containers } & \multirow{3}{*}{ Wet } & Outdoor & 20 & 14 & $34(97.1)$ & \multirow{3}{*}{$<0.001^{*}$} \\
\hline & & Indoor & 0 & 1 & $1(2.9)$ & \\
\hline & & Total & $20(57.1)$ & 15 (42.9) & 35 & \\
\hline & \multirow{3}{*}{ Dry } & Outdoor & 27 & 17 & $44(100)$ & \multirow{3}{*}{ NA } \\
\hline & & Indoor & 0 & 0 & 0 & \\
\hline & & Total & $27(61.4)$ & $17(38.4)$ & 44 & \\
\hline
\end{tabular}

NA: not applicable.

* Statistically significant with McNemar's test for two-independent samples. 
TABLE 3: Seasonal and geographical distributions of Aedes vectors at household level.

\begin{tabular}{|c|c|c|c|c|c|}
\hline \multirow[t]{2}{*}{ Season } & \multirow{2}{*}{$\begin{array}{l}\text { Households infested } \\
\text { with Aedes vectors }\end{array}$} & \multicolumn{3}{|c|}{$\begin{array}{c}\text { Number (\%) of households surveyed between the study } \\
\text { settings }\end{array}$} & \multirow[t]{2}{*}{$P$ value } \\
\hline & & Urban & Rural & Total & \\
\hline \multirow{3}{*}{ Wet } & Yes & 80 & 86 & $166(55.3)$ & \multirow{3}{*}{0.238} \\
\hline & No & 70 & 64 & $134(44.7)$ & \\
\hline & Total & $150(50.0)$ & $150(50.0)$ & 300 & \\
\hline \multirow{3}{*}{ Dry } & Yes & 94 & 84 & $178(59.3)$ & \multirow{3}{*}{$0.022^{*}$} \\
\hline & No & 56 & 66 & $122(40.7)$ & \\
\hline & Total & $150(50.0)$ & $150(50.0)$ & 300 & \\
\hline
\end{tabular}

${ }^{*}$ Statistically significant with McNemar's test for two-independent samples.

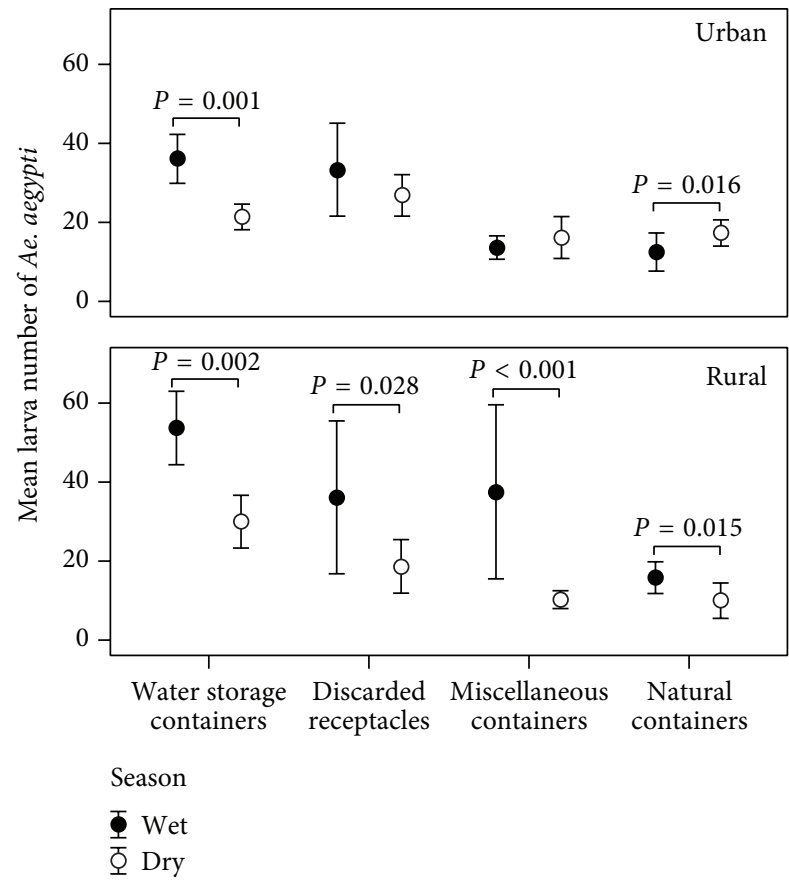

(a)

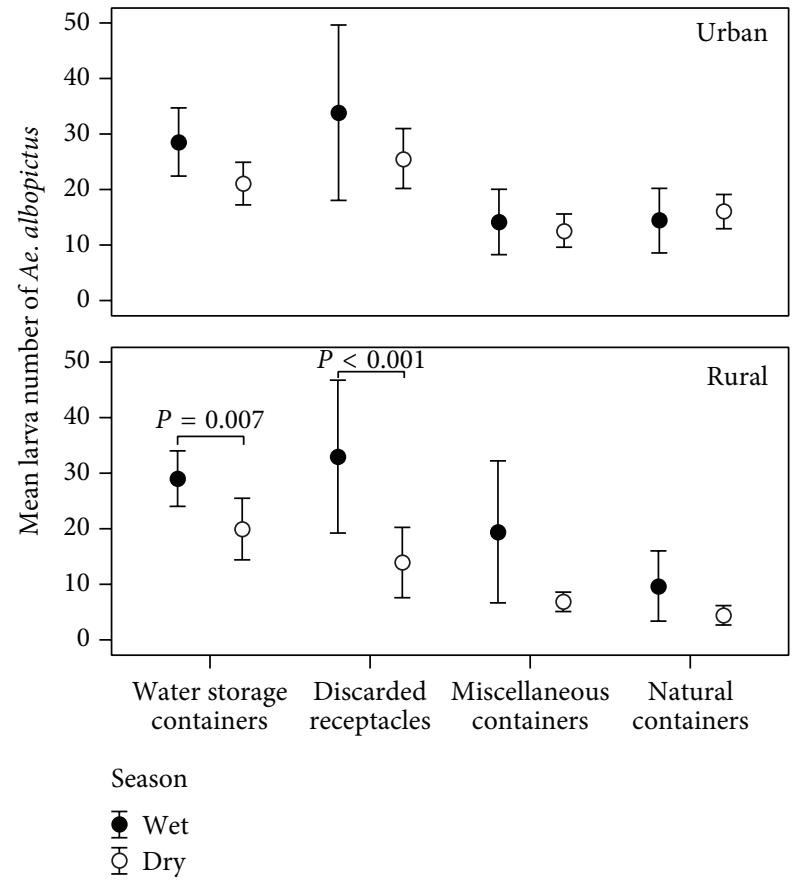

(b)

FIgURE 3: Comparisons of the abundance of Ae. aegypti and Ae. albopictus in each container type by season. The abundance of Aedes vectors was presented by mean larva numbers ( $\pm 2 \mathrm{SE}$ ) of Ae. aegypti (a) and Ae. albopictus (b) as denoted by error bars. Only the outdoor distributions of Aedes larva numbers in any key containers were further analyzed by the independent observations between two seasons in two study settings. In the urban-rural gradient, the equal distributions of Aedes larvae independently observed between seasons were tested using the Mann-Whitney $U$ test; the only two-independent samples that provided statistically significance $(P<0.05)$ are shown.

3.2. Household as the Unit of Analysis. Table 3 shows the temporal and spatial distributions of Aedes vectors that infested households in the urban $(n=150)$ and rural $(n=$ 150) settings, regardless of the kind of Aedes vectors. There was significant difference in the proportions of households infested with Aedes vectors between the urban and rural settings $(P=0.022)$ when analyzed for only the independent observation by dry season. When the Aedes infestation was compared between the urban and rural climates, only the Aedes-infested households that have Aedes larva-producing containers were considered (Figure 4$)$. The urban setting $(n=150)$ included $80(53.3 \%)$ and 94 (62.7\%) Aedes-infested households observed between wet and dry seasons. The rural setting $(n=150)$ included $86(57.3 \%)$ and $84(56.0 \%)$ Aedesinfested households independently observed between wet and dry seasons. On the other hand, the distributions of mean CI values in dry season were significantly lower than that in other wet seasons; for the urban setting, $Z=-2.125$, $P=0.034$; for the rural setting, $Z=-3.246, P=0.001$ (Figure 4).

\section{Discussion}

Like other receptive areas of Southeast Asia including Thailand [6, 8, 9], Philippines [7], and Malaysia [13, 28], Narathiwat bordered by Malaysia has experienced the land use and 


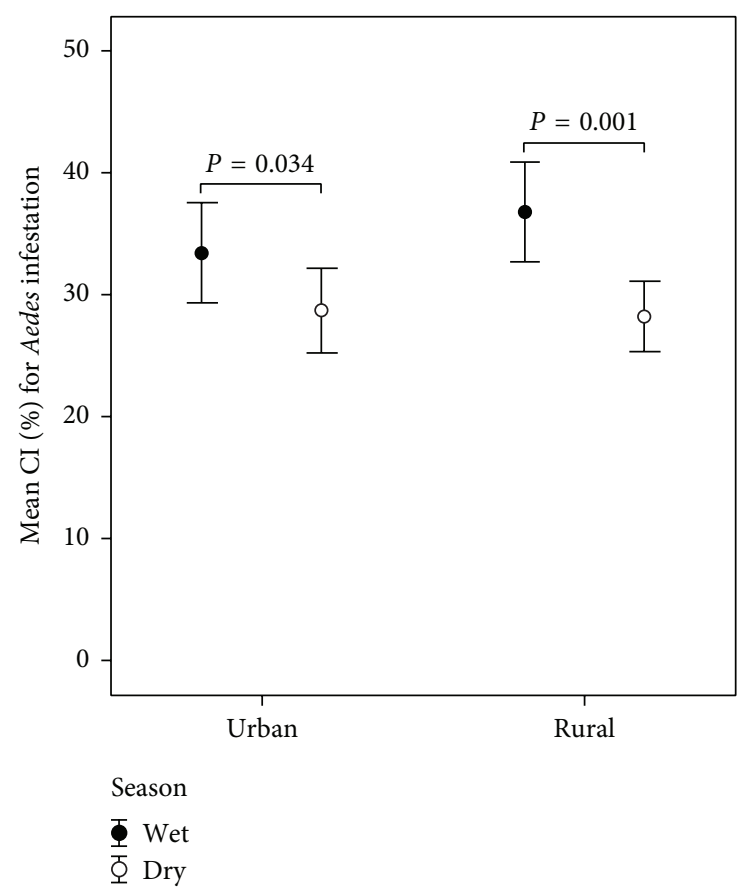

FIgURE 4: Comparisons of the Aedes-producing containers in the infested households of the urban and rural settings by season. Mean percentages $( \pm 2 \mathrm{SE})$ of $\mathrm{CI}$ values as denoted by error bars were presented between two study settings. The statistical significance with the Mann-Whitney $U$ test $(P<0.05)$ is shown for two categorical seasons on the distributions of Aedes larva numbers.

land cover change, although linked with diverse spatiotemporal distributions of Aedes vectors. The spatiotemporal distribution of dengue vectors like Ae. aegypti and Ae. albopictus that can infest or reinfest artificial and natural water-holding containers in the impoverished human inhabitations is often indirect and dynamic over space and time. In present study, it was clear to note that the artificial containers served as key water-holding containers available for Aedes breeding in the urban and rural settings. During wet season, the rural setting produced more artificial containers available for indoor rather than outdoor breeding of Aedes vectors. During dry season, there was tendency of the availability of more artificial containers in both urban and rural settings, but the outdoor and indoor distributions were similar. As for the natural container type, there was tendency of more natural containers available for outdoor breeding of Aedes vectors. To explore what breeding characteristics of Aedes vectors are, it was clear that, as seen in Table 2 and Figure 3, Aedes vectors produced the offspring by breeding outdoors rather than indoors in all 4 container types available during wet season rather than during dry season. Hence, spatiotemporal distributions of more artificial containers might contribute substantially to the greater variability of larval abundances (per container type) and container index (per house of each study setting) between urban and rural settings.

Water storage containers (especially jars, tanks, bath basins, and drums) $[6-8,12,28]$ were more likely to be favorable for Aedes vectors to breed outdoors in both wet and dry seasons. However, the spatiotemporal distributions of Aedes vectors that infested in water storage containers were not proportional to size, even though their indoor and outdoor distributions were similar for both seasons (data not shown). It was not surprising that the people residing in rural rather than urban setting-whether or not they had access to piped water-stored rainwater or other water sources for domestic use in as many as jars, tanks, bath basins, or drums during wet season. This is because there is tendency of shortage of water supply during dry season as seen in Figure 2. Moreover, many Aedes-infested water storage containers found in this study were laid outdoors or under the roof and were not covered with any cover types. In wet season, rural setting yielded outdoors versus indoors larval abundances, 21.4 versus $3.6 \mathrm{Ae}$. aegypti larvae and 12.2 versus $2.0 \mathrm{Ae}$. albopictus larvae per water storage container. Urban setting yielded outdoors versus indoors larval abundances, 14.4 versus 0.6 Ae. aegypti larvae and 11.6 versus $0.1 \mathrm{Ae}$. albopictus larvae per water storage container. In dry season, rural setting yielded outdoors versus indoors larval abundances, 9.2 versus 0.9 Ae. aegypti larvae and 5.2 versus 0.9 Ae. albopictus larvae per water storage container. Urban setting yielded outdoors versus indoors larval abundances, 4.8 versus 0.9 Ae. aegypti larvae and 4.8 versus $0.5 \mathrm{Ae}$. albopictus larvae per water storage container.

Miscellaneous containers (especially saucers, cabinet ant traps, and flowerpots) [6-8] were also favorable to breed Aedes vectors indoors rather than outdoors. Unlike water storage container type, the miscellaneous containers infested with Aedes vectors were proportional to size for both seasons and study settings (data not shown). This was because many households from both urban and rural settings produced household decorating items or accessories that were abandoned indoors rather than outdoors and often became water-holding containers that produced larval abundances. However, when examined for larval abundances, the outdoor breeding for Aedes vectors showed the densities greater than the indoor breeding. In wet season, rural setting yielded outdoors versus indoors larval abundances, 21.8 versus $3.1 \mathrm{Ae}$. aegypti larvae and 10.2 versus 1.1 Ae. albopictus larvae per miscellaneous container. Urban setting yielded outdoors versus indoors larval abundances, 3.5 versus 1.0 Ae. aegypti larvae and 4.3 versus $0.7 \mathrm{Ae}$. albopictus larvae per miscellaneous container. In dry season, urban setting yielded outdoors versus indoors larval abundances, 6.2 versus $1.2 \mathrm{Ae}$. aegypti larvae and 4.4 versus 1.1 Ae. albopictus larvae per miscellaneous container. Rural setting also yielded outdoors versus indoors larval abundances, 4.1 versus 0.6 Ae. aegypti larvae and 2.2 versus $0.3 \mathrm{Ae}$. albopictus larvae per miscellaneous container.

Discarded receptacles (especially used tyres, buckets, and ice bins) $[6-8,11,29]$ were likely to be favorable for Aedes vectors to breed outdoors. Many households from both urban and rural settings produced household wastes that were abandoned outdoors and often became water-holding containers as potential breeding places for Aedes vectors. In wet season, rural setting yielded outdoors versus indoors larval abundances, 10.0 versus $3.6 \mathrm{Ae}$. aegypti larvae and 10.4 versus $2.0 \mathrm{Ae}$. albopictus larvae per discarded receptacle. 
Urban setting yielded only outdoors larval abundances, 6.6 Ae. aegypti larvae and 6.7 Ae. albopictus larvae per discard receptacle. In dry season, urban setting yielded only outdoors larval abundances, 6.8 Ae. aegypti larvae and 6.0 Ae. albopictus larvae per discarded receptacle, whereas, in rural setting, outdoors larval abundances were 4.7 Ae. aegypti larvae and 3.2 Ae. albopictus larvae per discarded receptacle.

Natural containers (especially coconut shells) $[6-8,28]$ were also preferred outdoor breeding places for Aedes vectors. In wet season, rural setting yielded outdoors versus indoors larval abundances, 5.4 versus 10.0 Ae. aegypti larvae and 3.3 versus zero Ae. albopictus larvae per natural container. Urban setting yielded only outdoors larval abundances, 5.4 Ae. aegypti larvae and 5.9 Ae. albopictus larvae per natural container. In dry season, urban setting yielded only outdoors larval abundances, 3.6 Ae. aegypti larvae and 3.4 Ae. albopictus larvae per natural container, whereas, in rural setting, outdoors larval abundances were 2.9 Ae. aegypti larvae and 1.1 Ae. albopictus larvae per natural container.

Among the 4 container types, the water storage containers used by the households in the rural settings of Narathiwat were likely to show the preponderance of larval habitats of Aedes vectors in both wet and dry seasons. However, the outdoor breeding for Aedes vectors in all 4 container types was likely to produce larval abundances higher than the indoor breeding. When analyzed for the receptive setting that Aedes vectors can infest temporally, our findings demonstrated that both urban and rural settings seemed to have Aedes larva-producing containers in wet season rather than in dry season, as seen in Figure 4. When analyzed for the Aedes larval abundance, such the findings agreed with the rainfall data that Aedes larval abundances were related to monthly rainfall. The findings implied that there was greater availability of breeding places for Aedes vectors in wet season than in dry season. The seasonal variation was likely to regulate the abundance and distribution of Aedes vectors in Narathiwat. But there was greater variability of larval abundances of Ae. aegypti and Ae. albopictus in different container types due to the factors underlying water storage behaviors and garbage management. In other words, the aspect of the urban-rural division divided by PDR values at the district level might influence the delineation of urban (high degree of heterogeneous landscape) and rural (low degree of heterogeneous landscape) settings upon the cluster sampling. The urban-rural division of the studied districts may be influenced by the PDR threshold values among the cluster samples. House characteristics might become the underlying factor that influences Aedes larva-producing containers, but there seemed to be indistinguishable from each other in this study. This may be a reason why two study settings (urban versus rural) were likely to show the stability of Aedes infestation levels: $17.8 \%$ versus $21.1 \%$ CI per house in wet season and $18.0 \%$ versus $15.8 \%$ CI per house in dry season.

In addition, the effective and sustained dengue vector control in Thailand has focused radically on the householdlevel practices in environmental cleaning (PEC) practices through dengue prevention and control campaign as the part of the National Dengue Prevention and Control Program (NDCP). In each province implementing the NDCP, if expected to accompany the campaign activity and the adoption of other appropriately designed dengue vector control measures, the enhancement of the household-level PEC practices has been thought to reach the achievable targets at both village and subdistrict levels. The dengue prevention and control campaign is often operated before, during, or after the dengue outbreaks in order to ascertain that the conveyed messages can penetrate most households by making use of all media and channels. Nonetheless, there were no strict directions on whether all the implementing provinces determine the degree to which any households operate PEC practices routinely despite the fact that PEC practices guided by the levels of central, provincial, and local health sectors are expected to have the consequences of reducing the desired level of Aedes infestation (CI and $\mathrm{HI}$ ).

Evidently, this study provided the proof that the household-level PEC practiced by the households from both urban and rural settings of Narathiwat had the impacts on larval abundances of Ae. aegypti in 3 artificial container types and hence the containment of Aedes breeding places. For instance, the intermittent household-level PEC practices for both urban and rural settings contributed strongly to the significant reduction of outdoor larval abundances of Ae. aegypti that was remarkably shown in Figure 3 for water storage containers as well as miscellaneous containers. However, discarded receptacle was only the container type that the people paid no or less attention to perform householdlevel PEC practices. Although discarded receptacles yielded larva productivity lower than water storage container and miscellaneous container, there is however a need for dengue vector surveillance and control to address them as potential breeding sites, as well as to diminish or dispose them in timeliness manner [21,24]. The findings might imply that, if expected to reach the conveyed messages of householdlevel PEC practices, most households from both urban and rural settings played significant role in the containment of Aedes breeding places. However, the study could not provide the reason why some households neglected PEC practices because there exist as many as key containers that still had larval abundances and were not cleaned.

In summary, the seasonal and geographical variations of Aedes vectors were dynamic in nature. Less urbanization is also important for dengue transmission risk. As the result of the urban-rural gradient, the rural ecology of dengue also relates the seasonal distributions of Aedes vectors to dengue transmission dynamics. This is because there was greater availability of more container types as potential breeding sites, especially for Ae. aegypti that had more competitive advantage than Ae. albopictus and contributed greatly to produce larval abundances in spatial overlap. The study suggests that the higher urban-rural gradient, the greater the risk for seasonal and geographical distributions of Aedes vectors that persistently infest in 4 container types as potential breeding sites in human inhabitations and surroundings. To mitigate the dengue transmission risks, the effective and sustained household-level PEC practices should be applied to all receptive areas to reduce the intensity of larval abundances and hence contain Aedes breeding places at both village and subdistrict levels. Furthermore, like other receptive areas of 
Malaysia [13, 28, 30], Narathiwat experienced cobreeding of Ae. aegypti and Ae. albopictus in both urban and rural settings as this epidemiological implication for dengue/chikungunya virus transmission needs further investigation. Such anthropogenic land use and land cover change that contributes greatly to the health impacts of vector-borne diseases such as malaria [31] and dengue [32] also needs to be logically analyzed to determine the seasonal and geographical distributions of Aedes vectors.

\section{Competing Interests}

The authors declare that there are no competing interests regarding the publishing of this paper.

\section{Acknowledgments}

This work was supported by the Faculty of Science and Technology, Nakhon Si Thammarat Rajabhat University, Nakhon Si Thammarat. The authors acknowledged Associate Professor Dr. Mullica Jaroensutasinee and the entomological survey teams at the Faculty of Science and Technology, Nakhon Si Thammarat Rajabhat University, in conjunction with the Global Learning and Observation to Benefit the Environment (GLOBE) program, for their invaluable assistance during field trips and data entry.

\section{References}

[1] S. C. Tewari, V. Thenmozhi, C. R. Katholi, R. Manavalan, A. Munirathinam, and A. Gajanana, "Dengue vector prevalence and virus infection in a rural area in south India," Tropical Medicine and International Health, vol. 9, no. 4, pp. 499-507, 2004.

[2] C. M. Ríos-Velásquez, C. T. Codeço, N. A. Honório et al., "Distribution of dengue vectors in neighborhoods with different urbanization types of Manaus, state of Amazonas, Brazil," Memórias do Instituto Oswaldo Cruz, vol. 102, no. 5, pp. 617-623, 2007.

[3] B. Kamgang, J. Y. Happi, P. Boisier et al., "Geographic and ecological distribution of the dengue and chikungunya virus vectors Aedes aegypti and Aedes albopictus in three major Cameroonian towns," Medical and Veterinary Entomology, vol. 24, no. 2, pp. 132-141, 2010.

[4] Y. Higa, "Dengue vectors and their spatial distribution," Tropical Medicine and Health, vol. 39, supplement 4, pp. 17-27, 2011.

[5] S. L. Richards, S. L. Anderson, and B. W. Alto, "Vector competence of Aedes aegypti and Aedes albopictus (Diptera: Culicidae) for dengue virus in the Florida Keys," Journal of Medical Entomology, vol. 49, no. 4, pp. 942-946, 2012.

[6] T. Chareonviriyaphap, P. Akratanakul, S. Nettanomsak, and S. Huntamai, "Larval habitats and distribution patterns of Aedes aegypti (Linnaeus) and Aedes albopictus (Skuse), in Thailand," Southeast Asian Journal of Tropical Medicine and Public Health, vol. 34, no. 3, pp. 529-535, 2003.

[7] F. E. Edillo, N. D. Roble, and N. D. Otero II, "The key breeding sites by pupal survey for dengue mosquito vectors, Aedes aegypti (Linnaeus) and Aedes albopictus (Skuse), in Guba, Cebu City, Philippines," Southeast Asian Journal of Tropical Medicine and Public Health, vol. 43, no. 6, pp. 1365-1374, 2012.
[8] U. Thavara, A. Tawatsin, C. Chansang et al., "Larval occurrence, oviposition behavior and biting activity of potential mosquito vectors of dengue on Samui Island, Thailand," Journal of Vector Ecology, vol. 26, no. 2, pp. 172-180, 2001.

[9] D. Strickman and P. Kittayapong, "Dengue and its vectors in Thailand: introduction to the study and seasonal distribution of Aedes larvae," The American Journal of Tropical Medicine and Hygiene, vol. 67, no. 3, pp. 247-259, 2002.

[10] C. Chansang and P. Kittayapong, "Application of mosquito sampling count and geospatial methods to improve dengue vector surveillance," The American Journal of Tropical Medicine and Hygiene, vol. 77, no. 5, pp. 897-902, 2007.

[11] Y. Higa, N. T. Yen, H. Kawada, T. H. Son, N. T. Hoa, and M. Takagi, "Geographic distribution of Aedes aegypti and Aedes albopictus collected from used tires in Vietnam," Journal of the American Mosquito Control Association, vol. 26, no. 1, pp. 1-9, 2010.

[12] A. Hiscox, A. Kaye, K. Vongphayloth et al., "Risk factors for the presence of Aedes aegypti and Aedes albopictus in domestic water-holding containers in areas impacted by the Nam Theun 2 hydroelectric project, Laos," The American Journal of Tropical Medicine and Hygiene, vol. 88, no. 6, pp. 1070-1078, 2013.

[13] M. A. Roslan, A. Shafie, R. Ngui, Y. A. L. Lim, and W. Y. W. Sulaiman, "Vertical infestation of the dengue vectors Aedes aegypti and Aedes albopictus in apartments in Kuala Lumpur, Malaysia," Journal of the American Mosquito Control Association, vol. 29, no. 4, pp. 328-336, 2013.

[14] P. Kittayapong, S. Yoksan, U. Chansang, C. Chansang, and A. Bhumiratana, "Suppression of dengue transmission by application of integrated vector control strategies at sero-positive GIS-based foci," The American Journal of Tropical Medicine and Hygiene, vol. 78, no. 1, pp. 70-76, 2008.

[15] S. Thammapalo, V. Chongsuvivatwong, A. Geater, and M. Dueravee, "Environmental factors and incidence of dengue fever and dengue haemorrhagic fever in an urban area, Southern Thailand," Epidemiology and Infection, vol. 136, no. 1, pp. 135143, 2008.

[16] S. Thongrungkiat, L. Wasinpiyamongkol, P. Maneekan, S. Prummongkol, and Y. Samung, "Natural transovarial dengue virus infection rate in both sexes of dark and pale forms of Aedes aegypti from an urban area of Bangkok, Thailand," Southeast Asian Journal of Tropical Medicine and Public Health, vol. 43, no. 5, pp. 1146-1152, 2012.

[17] N. W. Beebe, R. D. Cooper, P. Mottram, and A. W. Sweeney, "Australia's dengue risk driven by human adaptation to climate change," PLoS Neglected Tropical Diseases, vol. 3, no. 5, article e429, 2009.

[18] S. Banu, W. Hu, C. Hurst, and S. Tong, "Dengue transmission in the Asia-Pacific region: impact of climate change and socioenvironmental factors," Tropical Medicine and International Health, vol. 16, no. 5, pp. 598-607, 2011.

[19] C. Åström, J. Rocklöv, S. Hales, A. Béguin, V. Louis, and R. Sauerborn, "Potential distribution of dengue fever under scenarios of climate change and economic development," EcoHealth, vol. 9, no. 4, pp. 448-454, 2012.

[20] F. J. Colón-González, C. Fezzi, I. R. Lake, and P. R. Hunter, “The effects of weather and climate change on dengue," PLoS Neglected Tropical Diseases, vol. 7, no. 11, Article ID e2503, 2013.

[21] A. Bhumiratana, A. Intarapuk, S. Chujun, W. Kaewwaen, P. Sorosjinda-Nunthawarasilp, and S. Koyadun, "Thailand momentum on policy and practice in local legislation on dengue vector 
control," Interdisciplinary Perspectives on Infectious Diseases, vol. 2014, Article ID 217237, 11 pages, 2014.

[22] P. Kittayapong, U. Chansang, C. Chansang, and A. Bhumiratana, "Community participation and appropriate technologies for dengue vector control at transmission foci in Thailand," Journal of the American Mosquito Control Association, vol. 22, no. 3, pp. 538-546, 2006.

[23] S. Koyadun, P. Butraporn, and P. Kittayapong, "Ecologic and sociodemographic risk determinants for dengue transmission in urban areas in Thailand," Interdisciplinary Perspectives on Infectious Diseases, vol. 2012, Article ID 907494, 12 pages, 2012.

[24] N. Suwannapong, M. Tipayamongkholgul, A. Bhumiratana, C. Boonshuyar, N. Howteerakul, and S. Poolthin, "Effect of community participation on household environment to mitigate dengue transmission in Thailand," Tropical Biomedicine, vol. 31, no. 1, pp. 149-158, 2014.

[25] B. H. B. van Benthem, S. O. Vanwambeke, N. Khantikul et al., "Spatial patterns of and risk factors for seropositivity for dengue infection," American Journal of Tropical Medicine and Hygiene, vol. 72, no. 2, pp. 201-208, 2005.

[26] S. O. Vanwambeke, B. H. B. van Bethem, N. Khantikul et al., "Multi-level analyses of spatial and temporal determinants for dengue infection," International Journal of Health Geographics, vol. 5, article 5, 2006.

[27] L. M. Ruedo, "Pictorial keys for the indentification of mosquitoes (Diptera: Culicidae) associated with dengue virus transmission," Zootaxa 589, Magnolia Press, Auckland, New Zealand, 2004.

[28] M. A. Nyamah, S. Sulaiman, and B. Omar, "Categorization of potential breeding sites of dengue vectors in Johor, Malaysia," Tropical Biomedicine, vol. 27, no. 1, pp. 33-40, 2010.

[29] S. Banerjee, G. Aditya, and G. K. Saha, "Household disposables as breeding habitats of dengue vectors: linking wastes and public health," Waste Management, vol. 33, no. 1, pp. 233-239, 2013.

[30] R. Norzahira, O. Hidayatulfathi, H. M. Wong et al., "Ovitrap surveillance of the dengue vectors, Aedes (Stegomyia) aegypti (L.) and Aedes (Stegomyia) albopictus skuse in selected areas in Bentong, Pahang, Malaysia," Tropical Biomedicine, vol. 28, no. 1, pp. 48-54, 2011.

[31] W. Kaewwaen and A. Bhumiratana, "Landscape ecology and epidemiology of malaria associated with rubber plantations in Thailand: integrated approaches to malaria ecotoping," Interdisciplinary Perspectives on Infectious Diseases, vol. 2015, Article ID 909106, 17 pages, 2015.

[32] Y. L. Cheong, P. J. Leitão, and T. Lakes, "Assessment of land use factors associated with dengue cases in Malaysia using boosted regression trees," Spatial and Spatio-temporal Epidemiology, vol. 10, pp. 75-84, 2014. 


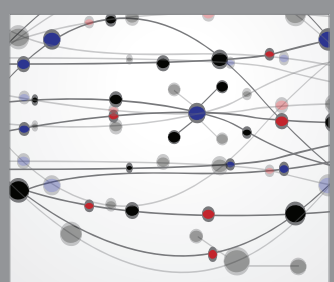

The Scientific World Journal
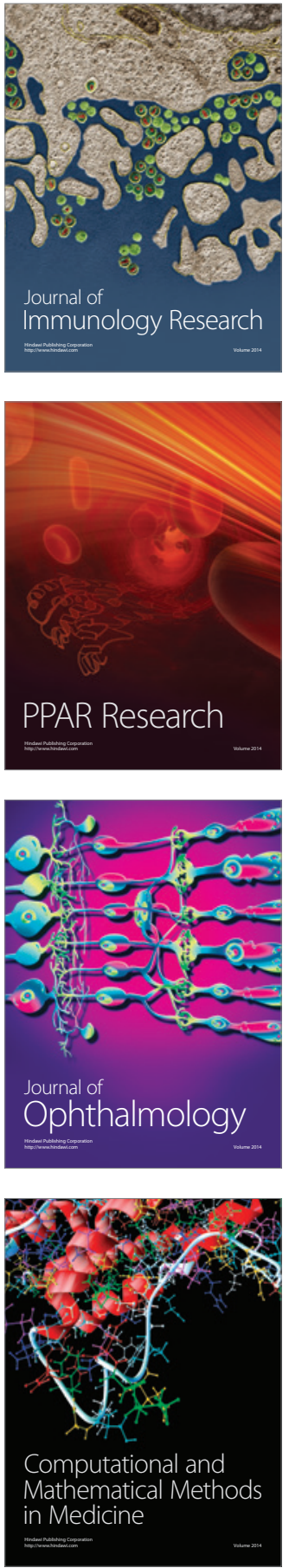

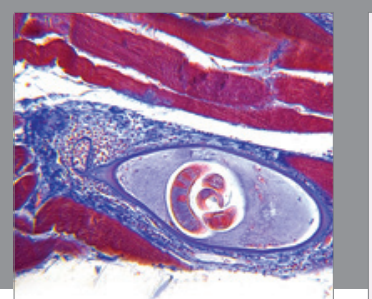

Gastroenterology Research and Practice

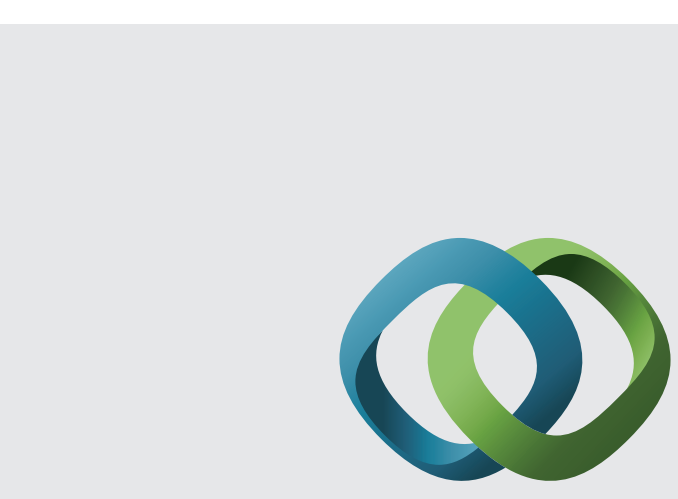

\section{Hindawi}

Submit your manuscripts at

http://www.hindawi.com
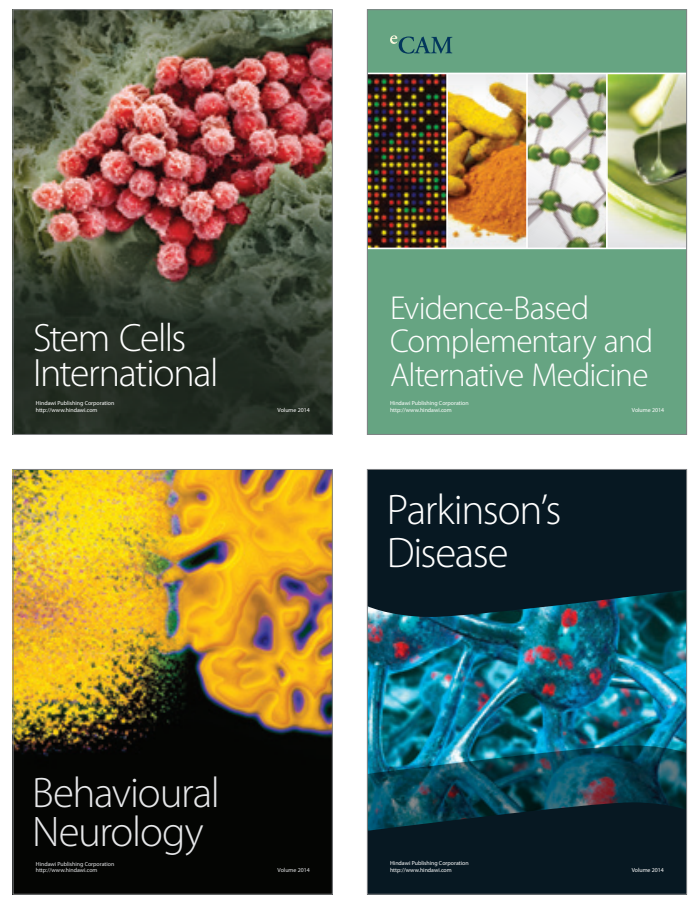
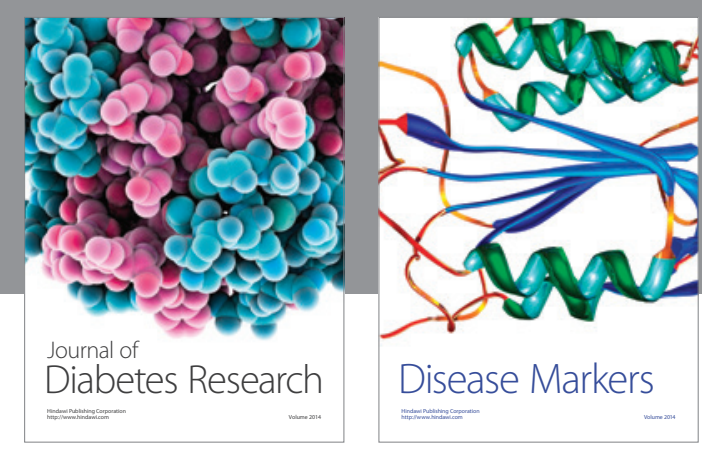

Disease Markers
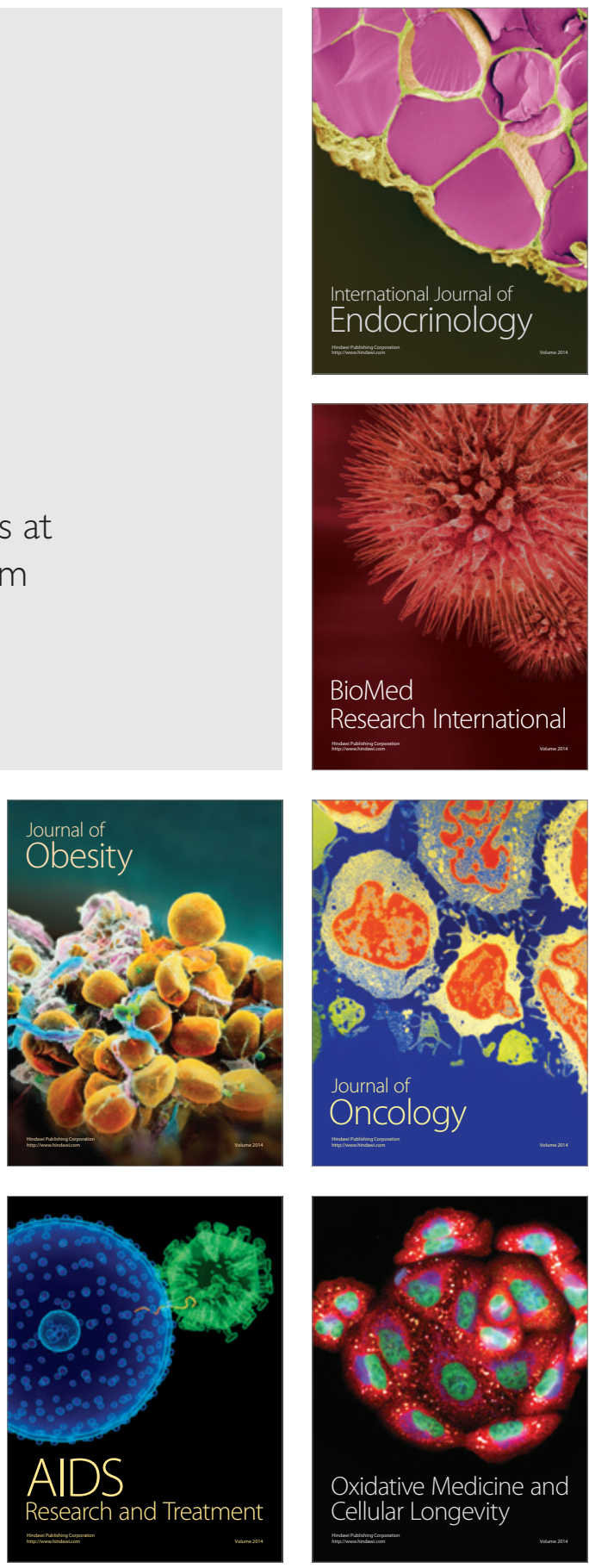